\title{
Uncertain fractional forward difference equations for Riemann-Liouville type
}

\author{
Qinyun Lu', Yuanguo Zhu' ${ }^{1 *}$ (D) and Ziqiang Lu
}

\section{"Correspondence:}

ygzhu@njust.edu.cn

${ }^{1}$ School of Science, Nanjing

University of Science and

Technology, Nanjing, China

\section{Springer}

\begin{abstract}
To model complex systems with discrete-time features and memory effects in the uncertain environment, a definition of an uncertain fractional forward difference equation with Riemann-Liouville-like forward difference is introduced. Moreover, analytic solutions to a type of special linear uncertain fractional difference equations are presented by the Picard iteration method. Then, an existence and uniqueness theorem of the solutions is proved by applying Banach contraction mapping theorem. Finally, two examples are provided to illustrate the validity of the existence and uniqueness theorem.
\end{abstract}

Keywords: Discrete fractional calculus; Uncertainty theory; Fractional forward difference equations

\section{Introduction}

Since 1965, fractional calculus has been a topic of interest and has become a useful tool for tackling problems in physics, biology, economics, and several fields in engineering [14]. In fact, due to the discrete-time features of these complex systems, some efforts have been made within discretization of continuous time methods. That is, numerical versions of continuous fractional calculus can result in the discrete models, but this treatment can readily lead to cumulate errors and cannot accurately depict the non-locality of complex systems. Consequently, research on discrete fractional calculus provides a very new idea to model the systems with memory effects and discrete-time features. In addition, with the improvement of computer technology, fractional difference equations are more easily solved by computers. Therefore fractional difference equations have been increasingly brought into focus. In this new area, a ground-breaking work has been set up by Miller and Ross. After that, the definitions and basic properties [5-7], correlation transformations [8], and equations [9-13] of fractional order difference have been studied in succession.

On the other hand, any system has its inherent structure and is disturbed by external factors in the practical application. Thereby, the fuzzy fractional differential equations (FFDEs) [14] and stochastic fractional differential equations (SSDEs) [15] have been extensively researched. A system disturbed by external objective random noises is popularly governed by SSDEs. This noise is usually characterized by Brownian motion. Nevertheless, when the system is interfered by external subjective noises, how to depict the subjective noises in mathematical language is a question. In general, external subjective noises

(c) The Author(s) 2019. This article is distributed under the terms of the Creative Commons Attribution 4.0 International License (http://creativecommons.org/licenses/by/4.0/), which permits unrestricted use, distribution, and reproduction in any medium, provided you give appropriate credit to the original author(s) and the source, provide a link to the Creative Commons license, and indicate if changes were made. 
make it difficult to accurately describe the behavior of systems. Fortunately, in 2007 [16] Liu established uncertainty theory for modeling belief degrees and refined it in 2010 [17] to do so. Then subjective noises may be described by canonical Liu process in the uncertainty theory. In recent years, more and more systems have been found to have memory effects such as stock price movement. To investigate systems with memory effects in the uncertain environment, the conception of uncertain fractional differential equations (UFDEs) rested on the uncertain theory was introduced in [18]. Furthermore, existence of their solutions was discussed in [19]. Recently, UFDEs were extended and applied to European option pricing in [20]. Meanwhile, comparison principles of the fractional differential equations were proposed in [21]. Furthermore, a numerical approach for solving UFDEs was also provided in [22] by the comparison principles in [21]. To our knowledge, research on the fractional equations in the uncertain environment is just beginning, and a lot of work needs to be done. So the uncertain fractional difference equations should be also explored to adjust to the need of theory and applications.

Motivated by the works mentioned above, to develop modeling techniques with discrete fractional calculus, we will define an uncertain fractional forward difference equation (UFFDE) and present an existence and uniqueness theorem of solutions to UFFDEs. The rest is arranged as follows: In Sect. 2, some facts in discrete fractional calculus are revisited. In Sect. 3, the fractional sum and difference for uncertain sequences are introduced. In Sect. 4, UFFDEs for Riemann-Liouville type are defined and the analytic solutions for a type of the linear UFFDEs for Riemann-Liouville type are obtained. In Sect. 5, an existence and uniqueness theorem of solutions is stated and verified. In Sect. 6, a conclusion is drawn.

\section{Preliminaries}

Some important facts in uncertainty theory, such as uncertainty space, uncertain measure, uncertain variable, and uncertainty distribution, may be adopted in the following sections. More details can be found in $[16,17,19]$. This section is concerned with some facts in fractional order sum. The $\mu$ th order fractional sum is defined in [8] as

$$
\Delta_{s}^{-\mu} f(t)=\frac{1}{\Gamma(\mu)} \sum_{l=s}^{t-\mu}(t-\sigma(l))^{(\mu-1)} f(l),
$$

where $\mu>0, s$ is a real number, $f(t)$ is a real-value function defined on $\mathbb{N}_{s}=\{s, s+1, \ldots\}$, $\Delta_{s}^{-\mu} f(t)$ is defined on $\mathbb{N}_{s+\mu}, \sigma(l)=l+1, t^{(\mu)}=\frac{\Gamma(t+1)}{\Gamma(t+1-\mu)}$, and $\Gamma(\mu)$ is the gamma function.

Note that the fractional sum $\Delta_{s}^{-\mu}$ maps functions whose domains are $\mathbb{N}_{s}$ to functions whose domains are $\mathbb{N}_{s+\mu}$. In the rest of this paper, the starting point $s$ is generally a real number.

An important result on the fractional sum operator will be presented.

Lemma 1 ([8]) Let $v \in R \backslash\{\ldots,-2,-1\}$, where $R$ represents the set of real numbers, $\mu>0$, and $t \in \mathbb{N}_{s+\mu+\nu}$. Then

$$
\Delta_{s+v}^{-\mu}(t-s)^{(v)}=\frac{\Gamma(v+1)}{\Gamma(\mu+1+v)}(t-s)^{(\mu+v)} .
$$




\section{Fractional sum and fractional Riemann-Liouville-like forward difference for uncertain sequences}

In this section, at first, the integer order difference and sum for uncertain sequences will be given. Then the fractional sum and the fractional Riemann-Liouville-like forward difference for uncertain sequences will be defined. The properties of the fractional sum operator for uncertain sequences will be finally obtained.

Definition 1 Let $h>0$ and $\xi_{t}$ be an uncertain sequence which is actually a series of uncertain variables indexed by $t=s+i h, i \in \mathbb{N}_{0}$. Then

$$
\Delta_{h} \xi_{t}=\frac{\xi_{t+h}-\xi_{t}}{h}
$$

is called first order forward difference of uncertain sequence $\xi_{t}$, where $h$ is a step size, and

$$
\Delta_{h}^{n} \xi_{t}:=\Delta_{h}\left(\Delta_{h}^{n-1} \xi_{t}\right)
$$

is called $n$th order forward difference of uncertain sequence $\xi_{t}$, where $n \geq 2$ is a natural number.

Definition 2 Let $h>0$ and $\xi_{t}$ be an uncertain sequence which is a series of uncertain variables indexed by $t=s+j h, j \in \mathbb{N}_{1}$. Then

$$
{ }_{s} \Delta_{h}^{-1} \xi_{t}=\sum_{l=s / h}^{t / h-1} \xi_{l h} h
$$

is called first order forward sum of uncertain sequence $\xi_{t}$, and

$$
{ }_{s} \Delta_{h}^{-n} \xi_{t}:={ }_{s} \Delta_{h}^{-1}\left({ }_{s} \Delta_{h}^{-(n-1)} \xi_{t}\right)
$$

is called $n$th order forward sum of uncertain sequence $\xi_{t}$, where $n \geq 2$ is a natural number.

Lemma 2 The following equality holds:

$$
\Delta_{h}\left({ }_{s} \Delta_{h}^{-1} \xi_{t}\right)=\xi_{t}
$$

Proof The equality is easily obtained by Definition 1 and Definition 2.

According to Eq. (1) and Eq. (2), we can readily obtain the positive integer $n$th order forward sum for uncertain sequence $\xi_{t}$ as follows:

$$
{ }_{s} \Delta_{h}^{-n} \xi_{t}=\frac{1}{\Gamma(n)} \sum_{l=s / h}^{t / h-n}(t-l h-h)_{h}^{(n-1)} \xi_{l h} h,
$$

where

$$
(t-l h-h)_{h}^{(n)}=(t-l h-h)(t-l h-2 h) \cdots(t-l h-n h)
$$


and $(t)_{h}^{(0)}=1$. Obviously, the right-hand side of formula (3) makes sense for all positive real number $n$. Generally, we consider the case with $h=1$. So we have a definition of any order sum of uncertain sequence as follows.

Definition 3 Assume that $\mu>0$ and $\xi_{t}$ is an uncertain sequence. Then

$$
\Delta_{s}^{-\mu} \xi_{t}=\frac{1}{\Gamma(\mu)} \sum_{l=s}^{t-\mu}(t-\sigma(l))^{(\mu-1)} \xi_{l}
$$

is called $\mu$ th order forward fractional sum for uncertain sequence $\xi_{t}$, where $t \in \mathbb{N}_{s+\mu}, \sigma(l)=$ $l+1$.

Definition 4 The fractional Riemann-Liouville-like forward difference for uncertain sequence $\xi_{t}$ is defined by

$$
\Delta_{s}^{v} \xi_{t}=\Delta^{q}\left(\Delta_{s}^{-(q-v)} \xi_{t}\right)
$$

where $v>0$ and $q-1<v \leq q, q$ represents a positive integer.

Lemma 3 Let $\mu, v>0$ and $\xi_{t}$ be an uncertain sequence. Then we have the following equalities:

$$
\Delta_{s+\mu}^{-v}\left(\Delta_{s}^{-\mu} \xi_{t}\right)=\Delta_{s}^{-(\mu+v)} \xi_{t}=\Delta_{s+\nu}^{-\mu}\left(\Delta_{s}^{-\nu} \xi_{t}\right)
$$

Proof According to the definition of fractional sum for uncertain sequences, conclusion is easy to be verified.

Lemma 4 For any real number $v>0$, we have

$$
\Delta_{s}^{-v} \Delta \xi_{t}=\Delta \Delta_{s}^{-v} \xi_{t}-\frac{(t-s)^{(v-1)}}{\Gamma(v)} \xi_{s}
$$

where $\xi_{t}$ is an uncertain sequence, and $\Delta \xi_{t}=\xi_{t+1}-\xi_{t}$.

Proof By similar derivation to [9], the lemma is easy to be verified.

4 Uncertain fractional forward difference equation for Riemann-Liouville type A definition of the UFFDE with Riemann-Liouville type will be exhibited in this section. The analytic solutions to a class of the linear UFFDEs with Riemann-Liouville type will be provided along with ones for linear uncertain first order forward difference equations.

Definition 5 A fractional difference equation is called an uncertain fractional difference equation if it is driven by an uncertain sequence. Further, an uncertain fractional forward difference equation for Riemann-Liouville type is the uncertain fractional difference equation with Riemann-Liouville-like forward difference. 
We will just explore the solution of the following UFFDE with the initial condition in this paper:

$$
\begin{aligned}
& \Delta_{\mu-1}^{\mu} Y(t)=G(t+\mu, Y(t+\mu))+H(t+\mu, Y(t+\mu)) \xi_{t+\mu}, \\
& \left.\Delta_{\mu-1}^{\mu-1} Y(t)\right|_{t=0}=Y_{0}
\end{aligned}
$$

where $\Delta_{\mu-1}^{\mu}$ denotes fractional Riemann-Liouville-like forward difference, $G, H:[0, \infty) \times$ $R \rightarrow R$ are two functions. $t \in \mathbb{N}_{0} \cap[0, T], \mu \in(0,1], Y_{0}$ is a crisp number, and $\xi_{\mu}, \xi_{\mu+1}, \ldots$, $\xi_{\mu+t}$ are $t+1$ i.i.d. uncertain variables that have linear uncertainty distribution $\mathcal{L}(a, b)$. A solution of UFFDE (5) with the initial condition (6) is an uncertain sequence $Y(t)$ that satisfies Eq. (5) uniformly in $t$.

Note that i.i.d. uncertain variables means that they are independent and have the same uncertainty distribution. More details can be seen in [17].

Remark 1 According to Definition 3 and Lemma 4, UFFDE (5) is equivalent to the uncertain fractional sum equation

$$
\begin{aligned}
Y(t)= & \frac{1}{\Gamma(\mu)} \sum_{l=0}^{t-\mu}(t-\sigma(l))^{(\mu-1)}\left(G(l+\mu, Y(l+\mu))+H(l+\mu, Y(l+\mu)) \xi_{l+\mu}\right) \\
& +\frac{Y_{0}}{\Gamma(\mu)} t^{(\mu-1)}
\end{aligned}
$$

for $t \in \mathbb{N}_{\mu} \cap[0, T]$.

The following special linear UFFDE will be considered in the sequel:

$$
\begin{aligned}
& \Delta_{\mu-1}^{\mu} Y(t)=\lambda Y(t+\mu)+\lambda \xi_{t+\mu}, \\
& \left.\Delta_{\mu-1}^{\mu-1} Y(t)\right|_{t=0}=Y_{0}
\end{aligned}
$$

for $t \in \mathbb{N}_{0} \cap[0, T]$, where $\Delta_{\mu-1}^{\mu}$ denotes the fractional Riemann-Liouville-like forward difference, $\mu \in(0,1], 0<\lambda<1, \xi_{\mu}, \xi_{\mu+1}, \ldots, \xi_{\mu+t}$ are $t+1$ i.i.d. uncertain variables that have linear uncertainty distribution $\mathcal{L}(a, b), Y_{0}$ is a crisp number.

Theorem 1 UFFDE (7) with the initial value condition (8) has a solution

$$
Y(t)=Y_{0} F_{\mu, \lambda}(t)+\zeta_{t}, \quad t \in \mathbb{N}_{\mu} \cap[0, T]
$$

for $0<\lambda<1$, where $\zeta_{t}$ is an uncertain sequence with the linear uncertainty distribution $\mathcal{L}\left(a \cdot e_{\mu, \lambda}(t), b \cdot e_{\mu, \lambda}(t)\right)$, and

$$
F_{\mu, \lambda}(t)=\sum_{k=0}^{\infty} \lambda^{k} \frac{(t+k \mu-1)^{((k+1) \mu-1)}}{\Gamma((k+1) \mu)},
$$

and

$$
e_{\mu, \lambda}(t)=\sum_{k=1}^{\infty} \lambda^{k} \frac{(t+(k-1) \mu)^{(k \mu)}}{\Gamma(k \mu+1)} .
$$


Proof The Picard approximation can be adopted to derive the solution. First, apply the $\Delta_{0}^{-\mu}=\Delta^{-\mu}$ operator to Eq. (7) to get

$$
\Delta^{-\mu} \Delta_{\mu-1}^{\mu} Y(t)=\lambda \Delta^{-\mu} Y(t+\mu)+\lambda \Delta^{-\mu} \xi_{t+\mu}, \quad t \in \mathbb{N}_{\mu} \cap[0, T] .
$$

Apply Lemma 2, Lemma 3, and Lemma 4 to the left-hand side of Eq. (12) to obtain

$$
\begin{aligned}
\Delta^{-\mu} \Delta_{\mu-1}^{\mu} Y(t) & =\Delta^{-\mu} \Delta \Delta_{\mu-1}^{-(1-\mu)} Y(t) \\
& =\Delta \Delta^{-\mu} \Delta_{\mu-1}^{-(1-\mu)} Y(t)-\frac{t^{(\mu-1)}}{\Gamma(\mu)} Y_{0} \\
& =\Delta \Delta^{-1} Y(t)-\frac{t^{(\mu-1)}}{\Gamma(\mu)} Y_{0} \\
& =Y(t)-\frac{t^{(\mu-1)}}{\Gamma(\mu)} Y_{0}, \quad t \in \mathbb{N}_{\mu} \cap[0, T] .
\end{aligned}
$$

According to Eq. (12), we have

$$
Y(t)-\frac{t^{(\mu-1)}}{\Gamma(\mu)} Y_{0}=\lambda \Delta^{-\mu} Y(t+\mu)+\lambda \Delta^{-\mu} \xi_{t+\mu}
$$

That is, the solution of UFFDE (7) is the solution of the sum equation as follows:

$$
Y(t)=\frac{t^{(\mu-1)}}{\Gamma(\mu)} Y_{0}+\lambda \Delta^{-\mu} Y(t+\mu)+\lambda \Delta^{-\mu} \xi_{t+\mu}, \quad t \in \mathbb{N}_{\mu} \cap[0, T] .
$$

Define $Y_{0}(t)=\frac{t^{(\mu-1)}}{\Gamma(\mu)} Y_{0}$ for $t \in \mathbb{N}_{\mu-1} \cap[0, T]$ and

$$
Y_{n}(t)=\frac{t^{(\mu-1)}}{\Gamma(\mu)} Y_{0}+\lambda \Delta^{-\mu} Y_{n-1}(t+\mu)+\lambda \Delta^{-\mu} \xi_{t+\mu}, \quad t \in \mathbb{N}_{\mu} \cap[0, T], n \in \mathbb{N}_{0} .
$$

Since $\xi_{\mu}, \xi_{\mu+1}, \ldots, \xi_{\mu+t}$ are $t+1$ i.i.d. uncertain variables, write $\xi_{\mu+t}=\xi$ in distribution. By Lemma 1 and the fact (Theorem 1.21 [17]) that the linear combination of finite independent linear uncertain variables is a linear uncertain variable with positive linear combination coefficient, we get

$$
\begin{aligned}
Y_{1}(t)= & \frac{t^{(\mu-1)}}{\Gamma(\mu)} Y_{0}+\lambda \Delta^{-\mu} Y_{0}(t+\mu-1)+\lambda \Delta^{-\mu} \xi \\
= & \frac{t^{(\mu-1)}}{\Gamma(\mu)} Y_{0}+\lambda \frac{(t+\mu-1)^{(2 \mu-1)}}{\Gamma(2 \mu)} Y_{0}+\lambda \frac{t^{(\mu)}}{\Gamma(\mu+1)} \xi, \\
Y_{2}(t)= & \frac{t^{(\mu-1)}}{\Gamma(\mu)} Y_{0}+\lambda \Delta^{-\mu} Y_{1}(t+\mu)+\lambda \Delta^{-\mu} \xi_{t+\mu} \\
= & \frac{t^{(\mu-1)}}{\Gamma(\mu)} Y_{0}+\lambda \frac{(t+\mu-1)^{(2 \mu-1)}}{\Gamma(2 \mu)} Y_{0}+\lambda^{2} \frac{(t+2 \mu-1)^{(3 \mu-1)}}{\Gamma(3 \mu)} Y_{0} \\
& +\lambda \frac{t^{(\mu)}}{\Gamma(\mu+1)} \xi+\lambda^{2} \frac{(t+\mu)^{(2 \mu)}}{\Gamma(2 \mu+1)} \xi,
\end{aligned}
$$




$$
Y_{n}(t)=\sum_{k=1}^{n} \lambda^{k} \frac{(t+(k-1) \mu)^{(k \mu)}}{\Gamma(k \mu+1)} \xi+\sum_{k=0}^{n} \lambda^{k} \frac{(t+k \mu-1)^{((k+1) \mu-1)}}{\Gamma((k+1) \mu)} Y_{0}, \quad t \in \mathbb{N}_{\mu} \cap[0, T],
$$

$\vdots$

Since the series

$$
\sum_{k=0}^{\infty} \lambda^{k} \frac{(t+(k-1) \mu)^{(k \mu)}}{\Gamma(k \mu+1)}
$$

and

$$
\sum_{k=0}^{\infty} \lambda^{k} \frac{(t+k \mu-1)^{((k+1) \mu-1)}}{\Gamma((k+1) \mu)}
$$

are absolutely convergent for $|\lambda|<1$ by the d'Alembert ratio comparison test, the limitation $\lim _{n \rightarrow \infty} Y_{n}$ exists. Write $Y_{n}(t) \rightarrow \bar{Y}(t)$ as $n \rightarrow \infty$. We have

$$
\bar{Y}(t)=\sum_{k=1}^{\infty} \lambda^{k} \frac{(t+(k-1) \mu)^{(k \mu)}}{\Gamma(k \mu+1)} \xi+\sum_{k=0}^{\infty} \lambda^{k} \frac{(t+k \mu-1)^{((k+1) \mu-1)}}{\Gamma((k+1) \mu)} Y_{0}, \quad t \in \mathbb{N}_{\mu} \cap[0, T] .
$$

Taking limit on both sides of Eq. (14) yields

$$
\bar{Y}(t)=\frac{t^{(\mu-1)}}{\Gamma(\mu)} Y_{0}+\lambda \Delta^{-\mu} \bar{Y}(t+\mu)+\lambda \Delta^{-\mu} \xi_{t+\mu}, \quad t \in \mathbb{N}_{\mu} \cap[0, T], n \in \mathbb{N}_{0} .
$$

That is, $\bar{Y}(t)$ satisfies Eq. (13). Hence $\bar{Y}(t)$ is a solution of Eq. (7) with the initial value condition (8). The proof is completed.

The following corollary will give the solutions of linear uncertain first order forward difference equations in light of Theorem 1.

Corollary 1 Uncertain first order forward difference equation

$$
\begin{aligned}
& \Delta Y(t)=\alpha Y(t+1)+\alpha \xi_{t+1}, \quad t \in \mathbb{N}_{0} \cap[0, T], \\
& \left.Y(t)\right|_{t=0}=Y_{0}
\end{aligned}
$$

has a solution

$$
Y(t)=Y_{0} \frac{1}{(1-\alpha)^{t}}+\eta_{t}, \quad t \in \mathbb{N}_{1} \cap[0, T]
$$

for $0<\alpha<1$, where $\eta_{t}$ is an uncertain sequence with linear uncertainty distribution $\mathcal{L}\left(\frac{a}{(1-\alpha)^{t}}-a, \frac{b}{(1-\alpha)^{t}}-b\right)$.

Proof Since $\sum_{k=0}^{\infty} \alpha^{k} \frac{(t+k-1)^{(k)}}{\Gamma(k+1)}=\frac{1}{(1-\alpha)^{t}}$, uncertain first order forward difference equation (15) with initial value condition (16) has a solution $Y(t)=\frac{1}{(1-\alpha)^{t}} Y_{0}+\eta(t)$ by Theorem 1 , where $\eta(t)$ is an uncertain sequence with linear uncertainty distribution $\mathcal{L}\left(\frac{a}{(1-\alpha)^{t}}-\right.$ $\left.a, \frac{b}{(1-\alpha)^{t}}-b\right)$. The conclusion is proved. 


\section{Existence and uniqueness}

Analytic solution is provided only for a special linear UFFDE. To find out the conditions that UFFDEs have solutions, an existence and uniqueness theorem is provided in this section.

Theorem 2 (Existence and uniqueness) Assume that $G(t, x)$ and $H(t, x)$ satisfy the Lipschitz condition

$$
|G(t, x)-G(t, y)|+|H(t, x)-H(t, y)| \leq L|x-y|
$$

and there is a positive number $L$ that satisfies the following inequality:

$$
L<\frac{\Gamma(\mu+1) \Gamma(T+1-\mu)}{\Gamma(T+1)(1+Q)}
$$

where $Q=|a| \vee|b|$. Then UFFDE (5) with initial value condition (6) has a unique solution $Y(t)$ for $t \in \mathbb{N}_{\mu} \cap[0, T]$ almost surely.

Proof Let $l_{\mu}^{k}$ be the set of all finite real sequences $x=\{x(t)\}_{\mu}^{k}$ which has $k$ terms with the norm $\|x\|=\max _{t \in \mathbb{N}_{\mu} \cap[0, T]}|x(t)|$. It is evident that $\left(l_{\mu}^{k},\|\cdot\|\right)$ is a Banach space. For any $X_{t}, Y_{t} \in$ $l_{\mu}^{k}$, the operator $P$ is defined as follows:

$$
\begin{aligned}
P X_{t}= & \frac{1}{\Gamma(\mu)} \sum_{l=0}^{t-\mu}(t-\sigma(l))^{(\mu-1)}\left(G\left(l+\mu, X_{l+\mu}\right)+H\left(l+\mu, X_{l+\mu}\right) \xi_{l+\mu}\right) \\
& +\frac{Y_{0}}{\Gamma(\mu)} t^{(\mu-1)} .
\end{aligned}
$$

At each time $t$, since $\xi_{t}\left(t \in \mathbb{N}_{\mu} \cap[0, T]\right)$ is an uncertain variable who has linear uncertainty distribution $\mathcal{L}(a, b)$, we have $\mathcal{M}\left\{\left(\xi_{t}<a\right) \cup\left(\xi_{t}>b\right)\right\}=0$. For any given $\gamma \in \Gamma \backslash\left\{\left(\xi_{t}<a\right) \cup\right.$ $\left.\left(\xi_{t}>b\right)\right\},\left|\xi_{t}(\gamma)\right| \leq Q(Q=|a| \vee|b|)$ holds almost surely for $t \in \mathbb{N}_{\mu} \cap[0, T]$, where $\Gamma$ is the universal set on the uncertainty space. Then

$$
\begin{aligned}
&\left\|P X_{t}(\gamma)-P Y_{t}(\gamma)\right\| \\
&=\max _{t \in \mathbb{N}_{\mu} \cap[0, T]}\left|P X_{t}(\gamma)-P Y_{t}(\gamma)\right| \\
& \leq \frac{1}{\Gamma(\mu)} \max _{t \in \mathbb{N}_{\mu} \cap[0, T]} \sum_{l=0}^{t-\mu}(t-\sigma(l))^{(\mu-1)}\left(\left|G\left(l+\mu, X_{l+\mu}(\gamma)\right)-G\left(l+\mu, Y_{l+\mu}(\gamma)\right)\right|\right. \\
&\left.+\left|\left[H\left(l+\mu, X_{l+\mu}(\gamma)\right)-H\left(l+\mu, Y_{l+\mu}(\gamma)\right)\right] \xi_{l+\mu}(\gamma)\right|\right) \\
& \leq \frac{1}{\Gamma(\mu)} \max _{t \in \mathbb{N}_{\mu} \cap[0, T]} \sum_{l=0}^{t-\mu}(t-\sigma(l))^{(\mu-1)}\left(\left|G\left(l+\mu, X_{l+\mu}(\gamma)\right)-G\left(l+\mu, Y_{l+\mu}(\gamma)\right)\right|\right. \\
&\left.+Q\left|H\left(l+\mu, X_{l+\mu}(\gamma)\right)-H\left(l+\mu, Y_{l+\mu}(\gamma)\right)\right|\right) \\
& \leq L(1+Q) \max _{t \in \mathbb{N}_{\mu} \cap[0, T]} \frac{1}{\Gamma(\mu)} \sum_{l=0}^{t-\mu}(t-\sigma(l))^{(\mu-1)}\left|X_{l+\mu}(\gamma)-Y_{l+\mu}(\gamma)\right|
\end{aligned}
$$




$$
\begin{aligned}
& \leq L(1+Q)\left\|X_{t}(\gamma)-Y_{t}(\gamma)\right\| \max _{t \in \mathbb{N}_{\mu} \cap[0, T]} \frac{1}{\Gamma(\mu)} \sum_{l=0}^{t-\mu}(t-\sigma(l))^{(\mu-1)} \\
& =L(1+Q)\left\|X_{t}(\gamma)-Y_{t}(\gamma)\right\| \max _{t \in \mathbb{N}_{\mu} \cap[0, T]} \Delta_{0}^{-\mu} t^{(0)} \\
& =L(1+Q)\left\|X_{t}(\gamma)-Y_{t}(\gamma)\right\| \max _{t \in \mathbb{N}_{\mu} \cap[0, T]} \frac{1}{\Gamma(\mu+1)} t^{(\mu) \quad(\text { by Lemma } 1)} \\
& =L(1+Q)\left\|X_{t}(\gamma)-Y_{t}(\gamma)\right\| \frac{1}{\Gamma(\mu+1)} \max _{t \in \mathbb{N}_{\mu} \cap[0, T]} t^{(\mu)} \\
& \leq \frac{L(1+Q) T^{(\mu)}}{\Gamma(\mu+1)}\left\|X_{t}(\gamma)-Y_{t}(\gamma)\right\| .
\end{aligned}
$$

When $0<L<\frac{\Gamma(\mu+1) \Gamma(T+1-\mu)}{\Gamma(T+1)(1+Q)}, P$ is a contraction mapping in $l_{\mu}^{k}$ almost surely. Thus we obtain a unique fixed point $Y_{t}(\gamma)$ of $P$ in $l_{\mu}^{k}$ almost surely by the Banach contraction mapping theorem. Furthermore, $Y_{t}(\gamma)=\lim _{n \rightarrow \infty} Y_{t}^{n}(\gamma)$, where $Y_{t}^{n}(\gamma)=P\left(Y_{t}^{n-1}(\gamma)\right)$ for $Y_{t}^{0}(\gamma)=\frac{Y_{0}}{\Gamma(\mu)} t^{(\mu-1)}$.

For any given $t \in \mathbb{N}_{\mu} \cap[0, T]$, as $G$ and $H$ are Lipschitz continuous functions, the operator $P$ is measurable. Note that $Y_{t}^{0}(\gamma)$ is an uncertain variable, $Y_{t}^{1}, Y_{t}^{2}, \ldots, Y_{t}^{n}, \ldots$ are uncertain variables since a real-valued measurable function of uncertain variables is an uncertain variable by Theorem 1.10 in [17]. Thus $Y_{t}=\lim _{n \rightarrow \infty} Y_{t}^{n}$ is an uncertain variable by Theorem 3 in [19].

Hence UFFDE (5) with initial value condition (6) has a unique solution $Y_{t}$ for $t \in \mathbb{N}_{\mu} \cap$ $[0, T]$ almost surely.

Here are two examples to illustrate Theorem 2.

Example 1 Consider the following UFFDE:

$$
\Delta_{-\frac{1}{2}}^{\frac{1}{2}} Y(t)=\frac{\sin Y\left(t+\frac{1}{2}\right)}{50+\left(t+\frac{1}{2}\right)^{2}}+\xi_{t+\frac{1}{2}}, \quad t \in \mathbb{N}_{0} \cap[0,4],
$$

where $\xi_{\frac{1}{2}}, \xi_{\frac{1}{2}+1}, \ldots, \xi_{\frac{1}{2}+4}$ are 5 i.i.d. uncertain variables with linear uncertainty distribution $\mathcal{L}(-1,2)$.

It is easy to verify that

$$
|G(t, x)-G(t, y)|+|H(t, x)-H(t, y)| \leq \frac{1}{50}|x-y|
$$

and

$$
\frac{\Gamma\left(\frac{1}{2}+1\right) \Gamma\left(4+1-\frac{1}{2}\right)}{3 \Gamma(4+1)} \approx 0.143>\frac{1}{50}
$$

Then UFFDE (19) has a unique solution almost surely by Theorem 2.

Example 2 Consider the following UFFDE:

$$
\Delta_{-\frac{1}{4}}^{\frac{1}{4}} X(t)=0.025 X^{2}\left(t+\frac{1}{4}\right)+\xi_{t+\frac{1}{4}}, \quad t \in \mathbb{N}_{0} \cap[0,3]
$$


with $X_{0}=\frac{1}{2}$, where $\xi_{\frac{1}{4}}, \xi_{\frac{1}{4}+1}, \xi_{\frac{1}{4}+2}$ and $\xi_{\frac{1}{4}+3}$ are 4 i.i.d. uncertain variables with linear uncertainty distribution $\mathcal{L}(-1,3)$.

Since the solution of Eq. (20) is the solution of the following sum equation with $X_{0}=\frac{1}{2}$

$$
X(t)=\frac{t^{(-0.75)}}{\Gamma(0.25)} X_{0}+\frac{1}{\Gamma(0.25)} \sum_{l=0}^{t-0.25}(t-l-1)^{(-0.75)}\left(0.025 X^{2}(l+0.25)+\xi_{l+0.25}\right)
$$

we have $X(t) \in[-20,20]$. It is easy to verify that

$$
|G(t, x)-G(t, y)|+|H(t, x)-H(t, y)| \leq 0.025|x-y||x+y| \leq 0.1|x-y|
$$

for $x, y \in[-20,20]$ and

$$
\frac{\Gamma\left(\frac{1}{4}+1\right) \Gamma\left(3+1-\frac{1}{4}\right)}{4 \Gamma(3+1)} \approx 0.167>0.1
$$

Then UFFDE(20) has a unique solution almost surely by Theorem 2 .

\section{Conclusions}

We mainly give a definition of an UFFDE and analytic solutions to a type of linear UFFDEs. Furthermore, a Lipschitz condition with an upper bounded constant results in that there is a unique solution almost surely provided to an UFFDE. Our results pave the way for our future work that is to explore the stability analysis and applications of UFFDEs.

Funding

This work is supported by the National Natural Science Foundation of China (No. 61673011) and Postgraduate Research \& Practice Innovation Program of Jiangsu Province (Grant No. KYCX18 0371).

\section{Competing interests}

The authors declare that they have no competing interests.

\section{Authors' contributions}

All the authors contributed equally and significantly in writing this paper. All the authors read and approved the final manuscript.

\section{Publisher's Note}

Springer Nature remains neutral with regard to jurisdictional claims in published maps and institutional affiliations.

Received: 8 January 2019 Accepted: 10 April 2019 Published online: 23 April 2019

\section{References}

1. Norton, L.: Gompertzian model of human breast cancer growth. Cancer Res. 48, 7067-7071 (1988)

2. Magin, R.: Fractional Calculus in Bioengineering. Begell House, New York (2006)

3. Sabatier, J., Agrawal, O., Tenreiro, M.: In Fractional Calculus: Theoretical Developments and Applications in Physics and Engineering. Springer, Berlin (2007)

4. Jumarie, G.: Stock exchange fractional dynamics defined as fractional exponential growth driven by (usual) Gaussian white noise. Application to fractional Black-Scholes equations. Insur. Math. Econ. 42, 271-287 (2008)

5. Podlubny, I.: Matrix approach to discrete fractional calculus. J. Comput. Phys. 228, 3137-3153 (2009)

6. Anastassion, G.: Nabla discrete fractional calculus and nabla inequalities. Math. Comput. Model. 51, $562-571$ (2010)

7. Abdeljawad, T.: On Riemann and Caputo fractional difference. Appl. Math. Comput. 62, 1602-1611 (2011)

8. Atici, F., Eloe, P.: A transform method in discrete fractional calculus. Integral and Finite Differ. Inequal. Appl. 2, 165-176 (2007)

9. Atici, F., Eloe, P.: Initial value problems in discrete fractional calculus. Proc. Am. Math. Soc. 137, 981-989 (2009)

10. Atici, F., Sengul, S.: Modeling with discrete fractional equations. J. Math. Anal. Appl. 369, 1-9 (2010)

11. Abu-Saris, R., Al-Mdallal, Q.: On the asymptotic stability of linear system of fractional-order difference equations. Fract. Calc. Appl. Anal. 16(2), 613-629 (2013) 
12. Chen, F., Luo, X., Zhou, Y.: Existence results for nonlinear fractional difference equation. Adv. Differ. Equ. 2011(1), 1 (2011)

13. Zhang, L., Zhou, Y.: Existence and attractivity of solutions for fractional difference equations. Adv. Differ. Equ. 2018(191), 1 (2018)

14. Chehlabi, M., Allahviranloo, T.: Concreted solutions to fuzzy linear fractional differential equations. Appl. Soft Comput. 44, 108-116 (2016)

15. Kamrani, M.: Numerical solution of stochastic fractional differential equations. Numer. Algorithms 68(1), 81-93 (2015)

16. Liu, B.: Uncertainty Theory, 2nd edn. Springer, Berlin (2007)

17. Liu, B.: Uncertainty Theory: A Branch of Mathematics for Modeling Human Uncertainty. Springer, Berlin (2010)

18. Zhu, Y.: Uncertain fractional differential equations and an interest rate model. Math. Methods Appl. Sci. 38, 3359-3368 (2015)

19. Zhu, Y.: Existence and uniqueness of the solution to uncertain fractional differential equation. J. Uncertain. Anal. Appl. 3, 1-11 (2015)

20. Lu, Z., Yan, H., Zhu, Y.: European option pricing model based on uncertain fractional differential equation. Fuzzy Optim. Decis. Mak. (2018). https://doi.org/10.1007/s10700-018-9293-4

21. Lu, Z., Zhu, Y.: Comparison principles for fractional differential equations with the Caputo derivatives. Adv. Differ. Equ. 2018(237), 1 (2018)

22. Lu, Z., Zhu, Y.: Numerical approach for solution to an uncertain fractional differential equation. Appl. Math. Comput. 343, 137-148 (2019)

Submit your manuscript to a SpringerOpen ${ }^{\circ}$ journal and benefit from:

- Convenient online submission

- Rigorous peer review

- Open access: articles freely available online

- High visibility within the field

- Retaining the copyright to your article

Submit your next manuscript at $\boldsymbol{\nabla}$ springeropen.com 